\title{
MEASUREMENT OF THE RESISTIVE WALL MODE STABILITY IN A ROTATING PLASMA USING ACTIVE MHD SPECTROSCOPY
}

\author{
by \\ H. REIMERDES, M.S. CHU, A.M. GAROFALO, G.L. JACKSON, \\ R.J. LA HAYE, G.A. NAVRATIL, M. OKABAYASHI, J.T. SCOVILLE, \\ and E.J. STRAIT
}

FEBRUARY 2003 


\section{DISCLAIMER}

This report was prepared as an account of work sponsored by an agency of the United States Government. Neither the United States Government nor any agency thereof, nor any of their employees, makes any warranty, express or implied, or assumes any legal liability or responsibility for the accuracy, completeness, or usefulness of any information, apparatus, product, or process disclosed, or represents that its use would not infringe privately owned rights. Reference herein to any specific commercial product, process, or service by trade name, trademark, manufacturer, or otherwise, does not necessarily constitute or imply its endorsement, recommendation, or favoring by the United States Government or any agency thereof. The views and opinions of authors expressed herein do not necessarily state or reflect those of the United States Government or any agency thereof. 


\title{
MEASUREMENT OF THE RESISTIVE WALL MODE STABILITY IN A ROTATING PLASMA USING ACTIVE MHD SPECTROSCOPY
}

\author{
by

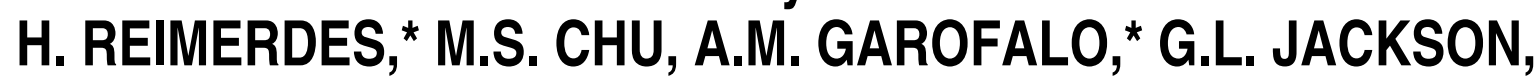 \\ R.J. LA HAYE, G.A. NAVRATIL, ${ }^{\star}$ M. OKABAYASHI, ${ }^{\dagger}$ J.T. SCOVILLE, \\ and E.J. STRAIT
}

This is a preprint of a paper to be submitted for publication in Phys. Rev. Lett.

*Columbia University, New York, New York.

†Princeton Plasma Physics Laboratory, Princeton, New Jersey.

\author{
Work supported by \\ the U.S. Department of Energy under \\ DE-FG03-89ER53297, DE-AC03-99ER54463, \\ and DE-AC02-76CH03073
}

GENERAL ATOMICS PROJECT 30200

FEBRUARY 2003 



\section{ABSTRACT}

The stability of the resistive-wall mode (RWM) in DIII-D plasmas above the conventional pressure limit, where toroidal plasma rotation in the order of a few percent of the Alfvén velocity is sufficient to stabilize the $n=1 \mathrm{RWM}$, has been probed using the technique of active MHD spectroscopy at frequencies of a few Hertz. The measured frequency spectrum of the plasma response to externally applied rotating resonant magnetic fields is well described by a single mode approach and provides an absolute measurement of the damping rate and the natural mode rotation frequency of the stable RWM. 

The maximum pressure in tokamak plasmas is often determined by the onset of a long-wavelength kink instability. In the presence of a resistive wall close to the plasma boundary, the ideal magnetohydrodynamic (MHD) free-boundary kink mode is converted into a slowly growing resistive-wall mode (RWM). The stabilization of the RWM could increase the operating regime of tokamaks from the no-wall up to the ideal wall stability limit. Operation above the no-wall limit is particularly important in advanced discharge scenarios, which aim at steady-state operation with a large fraction of bootstrap current. We report here on the first use of active, low-frequency "MHD spectroscopy" to study the stability properties of the RWM in tokamak plasmas where the ratio of plasma and magnetic pressure, $\beta=2 \mu_{0}\langle p\rangle / B_{0}^{2}$, exceeds the conventional, no-wall stability limit.

The present research pursues two different approaches in order to stabilize the RWM. In analogy to the control of the vertical instability in elongated tokamaks, feedback control using externally applied magnetic fields has been demonstrated in HBT-EP [1] and DIII-D [2,3]. Secondly, toroidal plasma rotation can stabilize the RWM due to an interaction between the quasi-static magnetic perturbation and the rapid plasma flow [4]. Sustained rotational stabilization has been demonstrated in DIII-D [5]. In the case of direct RWM feedback the correct description of the interaction of externally applied magnetic fields with the plasma is critical for the modeling of feedback performance. In this Letter we show that a single-mode approach satisfactorily describes the frequency response of the plasma to externally applied fields. In the second case the underlying dissipative process must be known in order to reliably predict the required rotation in a burning plasma experiment. Several mechanisms have been proposed and are discussed. In DIII-D the critical rotation frequency required for the stabilization of the RWM is a few percent of the inverse Alfvén time $\tau_{\mathrm{A}}=R_{0}\left(\mu_{0} \rho\right)^{1 / 2} / B_{0}$, where $R_{0}$ is the major radius, $B_{0}$ the toroidal magnetic field on axis, and $\rho$ the local mass density [6]. This is consistent with dissipation mechanisms based on coupling to sound waves and subsequent ion Landau damping [4] or kinetic damping [7], but it generally excludes dissipation models based on resistive MHD. The RWM stability has also been actively probed by measuring the plasma response to externally applied resonant magnetic field pulses $[8,9]$. Here, we generalize the measurement of the plasma response to externally applied fields by probing the entire relevant range of frequencies. This is an extension of the technique of active MHD spectroscopy, which was previously applied to MHD modes at frequencies above $10 \mathrm{kHz}$ [10], to frequencies of a few $\mathrm{Hz}$. 
A weakly damped mode, such as the RWM in a rotating plasma, can amplify the resonant component of magnetic field asymmetries, a phenomena referred to as resonant field amplification (RFA) [11]. RFA of externally applied pulsed magnetic fields using a set of six external picture-frame coils on the outboard midplane $(C$ coil $)$ has revealed that the plasma response has the same structure as the unstable RWM and that its amplitude increases significantly once the plasma pressure exceeds the no-wall limit [8]. The measurement of the perturbed radial magnetic field at the wall $B_{s}$ yields the RFA amplitude, which is defined as the ratio of the plasma response $B_{s}^{\text {plas }}=B_{s}-B_{s}^{\text {ext }}$ and the externally applied field $B_{s}^{\text {ext }}$ :

$$
A_{\mathrm{RFA}, s}=B_{s}^{\text {plas }} / B_{s}^{\mathrm{ext}},
$$

where the subscript $s$ indicates that these quantities implicitly include the coupling of the external field and plasma response to a particular array of sensors. Variables which exhibit the toroidal symmetry of the mode, such as the perturbation of the magnetic field at the wall $B_{s}(t)$ or currents in control coils $I_{\mathrm{c}}(t)$, are represented by a complex number so that $f(t, \varphi)=\Re\left|F(t) \cdot e^{-i n \varphi}\right|$. Thus, $A_{\mathrm{RFA}, s}$ is a complex quantity whose phase angle refers to the toroidal phase of the plasma response with respect to the externally applied field. Since the plasma response, which has the structure of the RWM, does not exactly match the structure of the externally applied field, the value of $A_{\mathrm{RFA}, s}$ depends on the geometry of the sensors and of the control coils.

The interaction of the RWM with external magnetic fields according to several singlemode models $[12,13]$ can be described by a system model for the evolution of the perturbed resonant radial magnetic field at the wall:

$$
\tau_{\mathrm{w}} \frac{d B_{s}}{d t}-\gamma_{0} \tau_{\mathrm{w}} B_{s}=M_{s c}^{*} I_{c}
$$

where $\gamma_{0}$ is the growth rate of the RWM in the absence of currents in any perturbing external coils $I_{c}$. Dissipation in a rotating plasma causes $\gamma_{0}$ to become a complex number. The real part of $\gamma_{0}$ describes the exponential growth or decay of the mode amplitude, while its imaginary part corresponds to the mode toroidal rotation in the lab frame. In the absence of plasma, $\gamma_{0}=-1 / \tau_{\mathrm{w}}$, where $\tau_{\mathrm{w}}$ is the characteristic resistive decay time for wall currents induced by the mode. An effective mutual inductance $M_{\mathrm{sc}}^{*}$ describes the direct coupling between the coils and the resonant component of the 
externally applied field at the wall and depends on the geometry of the coils and RWM mode structure. For a constant externally applied field ( $d / d t=0)$, Eq. (2) yields

$$
A_{\mathrm{RFA}, s}=c_{s} \frac{1+\gamma_{0} \tau_{\mathrm{w}}}{\left(-\gamma_{0} \tau_{\mathrm{w}}\right)},
$$

where $c_{s}=M_{s c}^{*} / M_{s c}$ is the ratio of the resonant component of the externally applied field to the externally applied field detected by the sensors $s$. The coefficient $c_{s}$ arises from the different spatial structure of the magnetic fields in the numerator and denominator of the definition of $A_{\mathrm{RFA}, s}$, Eq. (1). Once the external current is switched off, the perturbation amplitude decays according to $B_{s} \propto 1-\exp \left(\gamma_{0} t\right)$, if stable. In DIII-D, the measurement of the magnitude and phase of $A_{\mathrm{RFA}, s}$ for $C$ coil pulses yields a value of $\gamma_{0}$ which is found to be in good agreement with the observed decay time [8]. The dynamic response to externally applied pulsed perturbations is also used in HBT-EP [9], where it has been successfully compared to the Fitzpatrick-Aydemir RWM dispersion relation [14]. The experiment is now generalized by applying an external field with various angular frequencies $\omega_{\text {ext }}$. After an initial transient phase all quantities oscillate with the externally imposed frequency and Eq. (2) yields

$$
A_{\mathrm{RFA}, s}=c_{s} \frac{1+\gamma_{0} \tau_{\mathrm{w}}}{i \omega_{\mathrm{ext}} \tau_{\mathrm{w}} \pm \gamma_{0} \tau_{\mathrm{w}}} .
$$

According to Eq. (4) the frequency with the largest response corresponds to the natural rotation frequency of the RWM and the maximum amplitude increases as the plasma approaches marginal stability.

In the active MHD spectroscopy experiments on DIII-D, a new set of nonaxisymmetric coils is used as an antenna, Fig. 1. It consists of 12 internal picture-frame coils, which are arranged in toroidal arrays above and below the outboard midplane (I coil) [15]. The individual coils are phased to generate a magnetic field, which rotates with a frequency $f_{\text {ext }}=\omega_{\text {ext }} /(2 \pi)$. The spatial structure of the generated field largely overlaps the structure of the $n=1$ kink mode, which is generally the most unstable global mode. The magnetic perturbation caused by the antenna currents in the plasma is calculated to be $\delta B / B_{0} \approx 10^{-4}-10^{-3}$ and has only a small effect on transport and plasma rotation. The plasma response is detected with toroidal arrays of saddle loops, which measure the radial magnetic field above, on and below the outboard midplane, Fig. 1. Vacuum measurements of the frequency dependent externally applied field $B_{\mathrm{s}}^{\text {ext }}$ are used 
to extract the plasma response from the measured magnetic perturbation. A fit of the measured $B_{s}^{\text {ext }}$ to $B_{s}^{\text {ext }}\left(\omega_{\text {ext }}\right)=M_{s c} I_{c} /\left(1+i \omega_{\text {ext }} \tau_{\mathrm{w}}\right)$ obtained from Eq. (2) yields the estimate for $\tau_{\mathrm{w}}$ of $2.5 \mathrm{~ms}$.

The external field is applied in an ELMy $\mathrm{H}$-mode discharge. Tangential neutral beam injection raises the plasma pressure above the no-wall limit, Fig. 2(a) and causes the plasma to rotate, Fig. 2(b). Correction of the intrinsic error field using the $C$ coil maintains the rotation above the critical frequency required for rotational stabilization [5]. The stability limit is calculated for a typical equilibrium, which was reconstructed using internal measurements of the pressure and current density. By scaling the pressure profile the ideal MHD code DCON [16] yields a no wall limit of $\beta_{\mathrm{N}} \approx 2.1$, where $\beta_{\mathrm{N}}$ is the value of $\beta(\%)$ normalized on $I(\mathrm{MA}) /[a(\mathrm{~m}) B(\mathrm{~T})]$. As the discharge evolves, the limit of $\beta_{\mathrm{N}}$ scales approximately with the internal inductance.

The plasma stability is probed in multiple identical discharges at frequencies ranging from -40 to $+60 \mathrm{~Hz}$. The magnitude of the $n=1$ plasma response $B_{s}^{\text {plas }}$ is of the order of several Gauss and well above the noise level. It clearly peaks for an externally applied field rotating with 10 to $20 \mathrm{~Hz}$ in the direction of the plasma rotation, Fig. 3(a). Such a frequency corresponds to $25 \%-30 \%$ of the inverse wall time, which is in good agreement with the

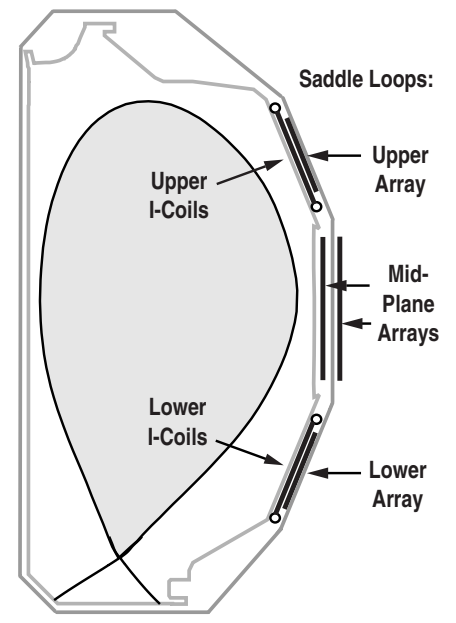

Fig. 1. Poloidal cross section of a plasma in DIII-D showing the location of the internal coils ( $I$ coil) used as an antenna and of various saddle loop arrays used as sensors.

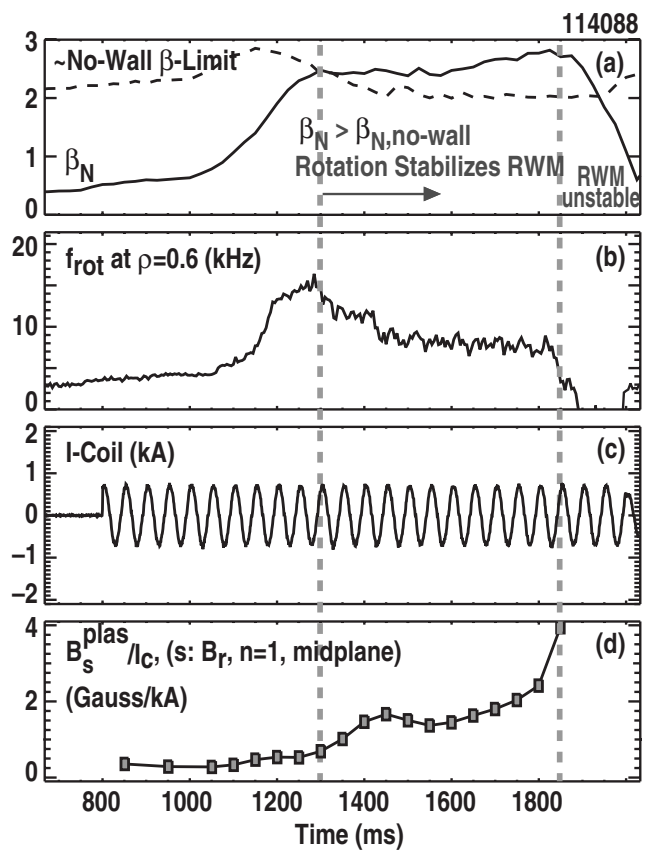

Fig. 2. (a) When $\beta_{\mathrm{N}}$ exceeds the no-wall stability limit $\beta_{\mathrm{N}, \text { no-wall }}$, (b) the plasma rotation frequency $f_{\text {rot }}$ is sufficiently large to stabilize the RWM. (c) The $I$ coil generates a rotating resonant field. (d) The $n=1$ plasma response $B_{s}^{\text {plas }}$ at $f=f_{\text {ext }}$ is measured with midplane saddle loops. 
predictions from the pulse experiments $[7,8]$. When $\beta_{\mathrm{N}}$ is increased from $2.4 \pm 0.1$ to $2.9 \pm 0.15$ the magnitude of $B_{s}^{\text {plas }}$ increases significantly. The frequency with the largest response, however, remains approximately constant. The poloidal structure of the plasma response is measured with saddle loop arrays at three poloidal locations. The difference of the toroidal phase of the $n=1$ component among the three arrays of approximately $120^{\circ}$, Fig. 3(b), is characteristic for the RWM and also observed for unstable modes [17]. This phase difference is independent of the applied frequency, i.e. the plasma response retains the same mode structure. The phase with respect to the externally applied field, however, changes with the applied frequency. While the plasma response leads the externally applied field for negative and small positive frequencies, it trails the externally applied field once the frequency is increased past the frequency of maximum amplitude, Fig. 3(b).

The measured frequency dependence of $A_{\mathrm{RFA}, s}$ is fitted to the predicted one-pole form Eq. (4). Fitting two free complex parameters, $\gamma_{0}$ and $c_{s}$, leads to good agreement with the measured RFA magnitude and phase, Fig. 4. The fit is equally good at both values of $\beta_{\mathrm{N}}$ and yields $\gamma_{0}=(-157+i 80) \mathrm{s}^{-1}$ at $\beta_{\mathrm{N}}=2.4$ and $\gamma_{0}=(-111+i 73) \mathrm{s}^{-1}$ at $\beta_{\mathrm{N}}=2.9$. The values of $c_{s}$ resulting from both fits are equal (within 10\%), consistent with a factor that depends only on the geometry of the sensors and the mode. Its magnitude is found to be 0.95 with a phase shift of $15^{\circ}$ in the direction of the plasma rotation. The phase shift may be caused by the up-down asymmetry of the single-null plasma. The good agreement between the measured spectrum and the fit shows that the interaction between the RWM 
and externally applied magnetic fields is well described by a single-mode model. Consequently, the fit parameter yields an absolute measurement of $\gamma_{0}$.

The measurement of $\gamma_{0}$ allows for a direct comparison with theory. A preliminary comparison demonstrating the feasibility of such a test of theory is shown in Fig. 5. Here, the measured values of $\gamma_{0}$ are compared to predictions of the MARS code [18] for sound wave damping [4] using a similar plasma, albeit with a flat rotation profile and an $L$-mode edge. In order to compare the

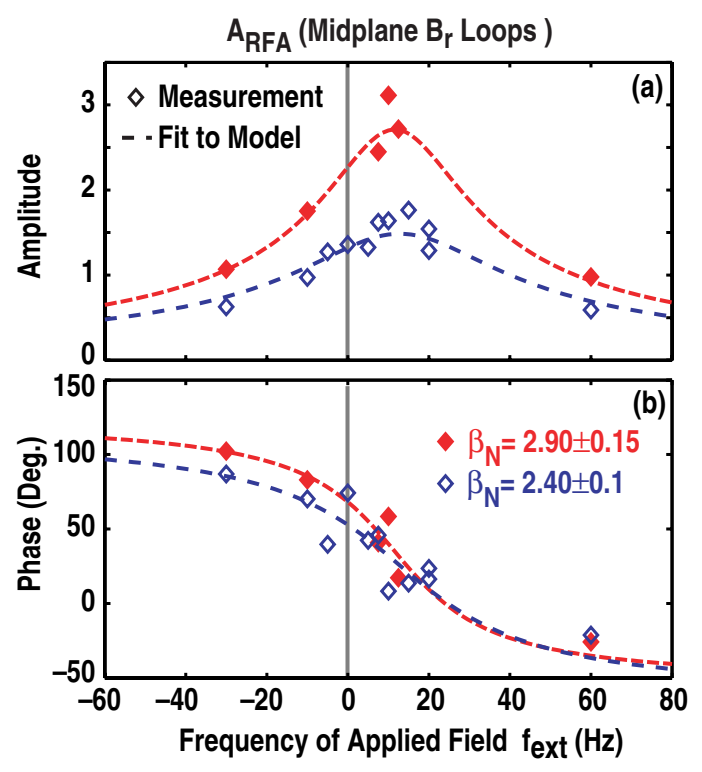

Fig. 4. The magnitude (a) and phase (b) of the $n=1 \quad A_{\mathrm{RFA}, s}$, Eq. (1), measured with midplane saddle loops are fitted to Eq. (4).

RWM growth rates, the gain in $\beta$ between the no-wall and ideal wall stability limit $C_{\beta}=\left(\beta-\beta_{\text {no-wall }}\right) /\left(\beta_{\text {ideal wall }}-\beta_{\text {no-wall }}\right)$ is used as the stability parameter. The parameter $\kappa_{\|}$ which accommodates toroidicity and shaping effects in the sound wave damping model is set to 0.5 . While the observed damping rate is in good agreement with the predictions for a plasma rotation frequency of $\omega_{\text {rot }} \tau_{\mathrm{A}}=2 \%$, Fig. $5(\mathrm{a})$, the observed mode rotation frequency is an order of magnitude lower than predicted, Fig. 5(b).

The active measurement can also provide a continuous measurement of the ideal MHD stability. The plasma response to the externally applied magnetic field in discharge 114088, Fig. 2, increases significantly once the pressure exceeds the estimated no-wall $\beta$-limit. At $t \approx 1800 \mathrm{~ms}$ the plasma rotation is not sufficient to stabilize the RWM and it becomes unstable. With $c_{s}$ known from the fit of the complete RFA spectrum, the measurement of the amplitude and phase at a single frequency is sufficient to calculate $\gamma_{0}$. The coherent detection technique allows for a small perturbation amplitude compared to pulsed techniques only trading off in time resolution which is limited to the period of the applied frequency. Such a measurement, carried out in real-time, is a potentially important input for profile control in an advanced tokamak, which relies on operation in a wall-stabilized regime. 

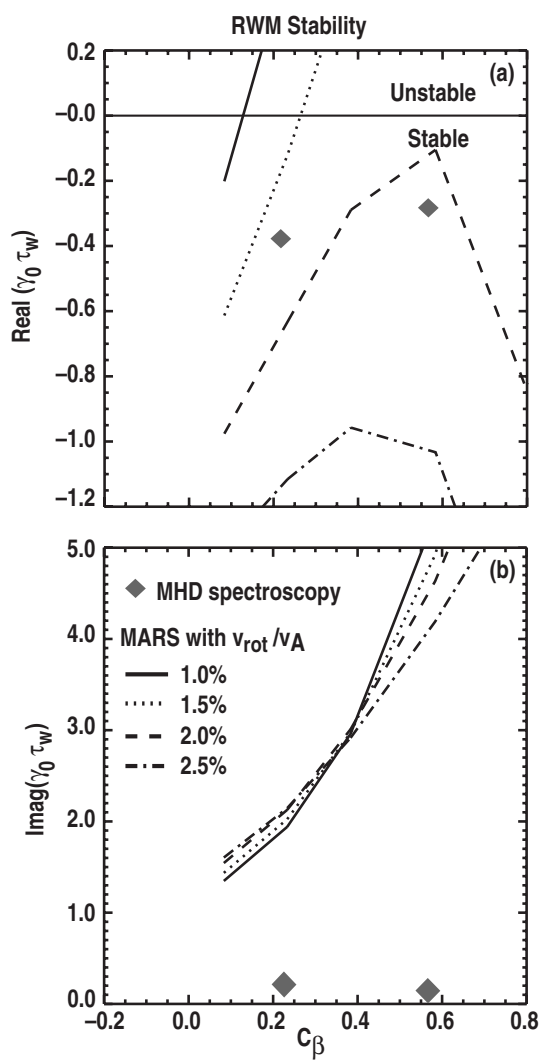

Fig. 5. Comparison of the measured real (a) and imaginary part (b) of $\gamma_{0}$ with MARS predictions for sound wave damping $\left(\kappa_{\|}=0.5\right)$ at several values of plasma rotation $\omega_{\text {rot }}$.

In conclusion, active measurements of the spectrum of the rotation stabilized RWM have been carried out. Rotating plasmas above the no-wall ideal MHD stability limit respond to externally applied slowly rotating magnetic fields. The structure of the plasma response is independent of the applied frequency and typical for an RWM. Maximum amplification is observed when the externally applied field rotates with a frequency of a fraction of the inverse wall time in the direction of the toroidal plasma rotation. The measured spectrum is well described by a single-mode model, which strongly supports the applicability of a single-mode approach to describe the interaction between externally applied fields and a marginally stable plasma. The fit of the measured spectrum yields an absolute measurement of the RWM damping rate in the absence of external fields, which allows a test of proposed dissipation mechanisms. The feasibility of such a comparison has been demonstrated by comparing the measurements to MARS predictions using the sound wave damping model. The measured spectrum also yields information about the geometrical coupling. With the coupling known, the measurement of the RFA at a single frequency can provide a continuous measurement of the global plasma stability, which can be an important input for profile control in an advanced tokamak. 
Work supported by U.S. Department of Energy under Contract Nos. DE-FG0389ER53297, DE-AC03-99ER54463, and DE-AC02-76CH03073.

[1] C. Cates et al., Phys. Plasmas 7, 3133 (2000).

[2] M. Okabayashi et al., Phys. Plasmas 8, 2071 (2002).

[3] E.J. Strait et al., Phys. Plasmas 11, 2505 (2004).

[4] A. Bondeson and D.J. Ward, Phys. Rev. Lett. 72, 2709 (1994).

[5] A.M. Garofalo et al., Phys. Rev. Lett. 89, 235001 (2002).

[6] R.J. La Haye, et al., Bull. Am. Phys. Soc. 47, 274 (2002).

[7] A. Bondeson and M.S. Chu, Phys. Plasmas 3, 3013 (1996).

[8] A.M. Garofalo, T.H. Jensen, and E.J. Strait, Phys. Plasmas 10, 4776 (2003).

[9] M. Shilov et al., Phys. Plasmas 11, 2573 (2004).

[10] A. Fasoli et al., Phys. Rev. Lett. 75, 645 (1995)

[11] A.H. Boozer, Phys. Rev. Lett. 86, 1176 (2001).

[12] A.M. Garofalo, T.H. Jensen, and E.J. Strait, Phys. Plasmas 9, 4573 (2002).

[13] M.S. Chu, et al., Nucl. Fusion 43, 196 (2003).

[14] R. Fitzpatrick, Phys. Plasmas 9, 3459 (2002).

[15] G.L. Jackson, et al., in Proceedings of the 30 ${ }^{\text {th }}$ EPS Conference on Plasma Physics and Controlled Fusion, St. Petersburg, Russia, 2003 (European Physical Society, Geneva, 2003), Vol. 27A, p. 4.47.

[16] A.H. Glasser and M.S. Chance, Bull. Am. Phys. Soc. 42, 1848 (1997).

[17] E.J. Strait, et al., Nucl. Fusion 39, 1977 (1999).

[18] Y.Q. Liu, et al., Phys. Plasmas 7, 3681 (2000). 